\section{Quand les clichés peuvent être évoqués}

\section{Bruno Kesseli}

Dr méd. et lic. phil., rédacteur en chef

C'est un aréopage complet de matière grise qui s'est rassemblé fin juin dernier à Valens, recoin haut perché de la vallée du Rhin. Cette région bucolique est célèbre pour ses gorges de la Tamina et ses thermes vieux de plusieurs siècles, dont Paracelse décrivait déjà les bienfaits thérapeutiques dans l'un de ses écrits. Si la renommée du centre de rééducation de Valens dépasse les frontières de la région et du pays, c'est avant tout grâce au médecin chef de son service de rééducation neurologique, le professeur Jürg Kesselring. Présent depuis 1987, il a fêté cette année son $30^{\mathrm{e}}$ anniversaire d'exercice à Valens.

Cette vie au cœur des Alpes n'a jamais été un obstacle pour lui, bien au contraire, et la liste de conférenciers du symposium montre qu'il a su lier et entretenir une multitude de relations professionnelles et culturelles en Suisse et tout autour du globe depuis sa terrasse à ciel ouvert de Valens. Reconnu comme l'un des grands pionniers dans le domaine de l'immunopathologie de la sclérose en plaques par le magazine Lancet en 2015, le Viennois Hans Lassmann a inauguré la rencontre, au cours de laquelle des neuroscientifiques comme Martin Schwab (Zurich) ou des neurologues comme Christian Hess, Claudio Bassetti (tous deux de Berne), Ludwig Kappos (Bâle) et Armin Schnider (Genève) ont apporté de nouveaux éclairages sur des sujets tels que la «Neurobiologie de la rééducation», la «Conscience», la «Neurobiologie du sommeil» ou encore la «Réalité et la mémoire».

Eckart Altenmüller, neurologue et musicien, a quant à lui fait vivre des instants aussi passionnants que joyeux avec son exposé sur «Le don d'Apollon: la musicothérapie neurologique dans la rééducation». Le Père Martin Werlen et Christine Beerli ont rappelé la variété des centres d'intérêt et l'engagement humanitaire, notamment auprès du Comité international de la Croix-Rouge (CICR), du protagoniste de cet anniversaire. L'ancien abbé du monastère d'Einsiedeln a fait part à son auditoire de ses réflexions sur «ce qui relie de manière surprenante la Foi et le Savoir», tandis que la vice-présidente du CICR a présenté son institution en l'abordant du point de vue de la "persistance d'une idée dans un monde qui change à toute allure».

Cet impressionnant symposium-anniversaire marquait en quelque sorte l'au revoir de Jürg Kesselring à la

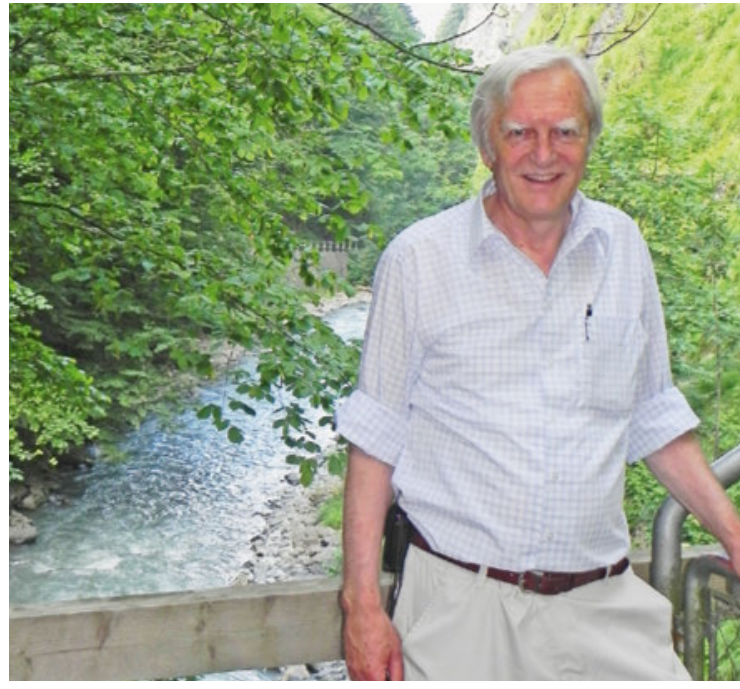

Esprit et espièglerie sur les chemins (ici dans les gorges de la Tamina): Jürg Kesselring.

clinique de Valens, qu'il a quittée en septembre dernier, non sans avoir organisé une fête avec ses collaborateurs. Ce départ à la retraite est l'occasion d'évoquer en toute bonne conscience deux clichés qui ont la peau dure. Il serait vain de vouloir évoquer les nombreuses activités et mérites de Jürg Kesselring dans un court article. Monsieur Kesselring n'était pas seulement un coryphée de la rééducation neurologique ayant pris en charge et accompagné des milliers de patients et s'étant investi dans d'innombrables comités d'experts et organisations. Il était et reste aussi un humaniste aux centres d'intérêts universels, qui, en plus de son activité médicale, s'est fait un nom en tant que musicien, essayiste et poète et s'est engagé dans l'humanitaire, notamment au CICR. Son attitude avenante et ses facultés de bâtisseur de ponts lui ont énormément facilité la tâche, ainsi qu'à ses interlocuteurs.

Le deuxième cliché en question est celui de la «retraite active». L'adjectif "active» est particulièrement bien choisi et s'accorde parfaitement avec d'autres attributs, comme intéressé, ouvert ou curieux, qui lui correspondent. Quoi qu'il en soit, une chose est sûre avec Jürg Kesselring: il continuera tout simplement à faire ce qui l'intéresse.

Quelques indices se profilent déjà. Je viens par exemple de recevoir sa réponse à un de mes courriels, avec la formule de conclusion suivante: «Meilleures salutations du plus grand congrès sur la sclérose en plaque qui se déroule à Paris avec 10000 participants, où je dois 'dire quelque chose' incessamment sous peu...»

Gageons que vous aurez bientôt le plaisir de lire un article sur Jürg Kesselring dans un prochain Bulletin des médecins suisses.

Crédit photo

Photo: Bruno Kesseli 